\title{
Improved patient outcomes by normalizing sympathovagal balance as measured by parasympathetic and sympathetic monitoring: the benefits of carvedilol
}

\author{
DePace $\mathrm{NL}^{1 *}$, Bateman $\mathrm{J}^{1}$, Yayac $\mathrm{M}^{1}$, Siddique $\mathrm{M}^{1}$, Pinales $\mathrm{J}^{1}$, Acosta $\mathrm{C}^{1}$ and Vinik $\mathrm{AI}^{2}$ \\ ${ }^{1}$ Hahnemann Hospital /Drexel University College of Medicine, Franklin Cardiovascular Associates, USA \\ ${ }^{2}$ Eastern Virginia Medical School, The Strelitz Diabetes Center, USA
}

\begin{abstract}
Autonomic Dysfunction (AD) is a progressive disorder that occurs naturally with age. Disease, injury, exposure, adverse lifestyle, etc., accelerate the autonomic aging process. Balancing the Parasympathetic and Sympathetic (P\&S) branches of the Autonomic Nervous System, slows the progression. Autonomic Neuropathy (AN) is the symptomatic phase of $\mathrm{AD}$, and includes increased morbidity and mortality risks, including risk of major adverse cardiovascular events such as stroke and heart attack. Balancing P\&S minimizes these risks. Carvedilol is a well-known beta-blocker with a (second) alpha-component. Carvedilol is often the medication of choice for patients diagnosed with diabetes and cardiovascular disease. It is well established that Diabetes significantly involves AD leading to AN, and Carvedilol seems to provide the best therapy, not only for minimizing cardiovascular risks, but for affecting a better P\&S balance. In a large cardiology cohort we compared the outcomes of patients prescribed standard beta-blocker therapy with matched patients prescribed Carvedilol. The actions of Carvedilol not only reduce Sympathetic activity, as do standard beta-blockers, but it also seems to promote a small but significant rebound in Parasympathetic activity. This rebound in Parasympathetic activity is cardioprotective. Carvedilol may be the therapy of choice in patients diagnosed with AN and cardiovascular disease, including for non-diabetic patients.
\end{abstract}

\section{Introduction}

Parasympathetic and Sympathetic (P\&S) Monitoring was introduced into a large cardiology cohort. Given the additional information from P\&S Monitoring, autonomic dysfunction was found to be more prevalent that previously known, especially in non-diabetic patients. Given the preference for Carvedilol in diabetic patients diagnosed with cardiovascular disease (CVD), Carvedilol was introduced for beta-blocker naïve patients or replace standard betablocker $(\beta B)$ therapy. Carvedilol has double efficacy with both a $\beta B$ and an alpha-blocker $(\alpha B)$. In the presence of autonomic neuropathy, the $\alpha \mathrm{B}$-combination seems to indirectly affect Parasympathetic $(\mathrm{P})$ activity. Other $\beta$ Bs do not include an $\alpha \mathrm{B}$ and (anecdotally) have not proven as effective as Carvedilol in autonomic patients with CVD [1].

Autonomic dysfunction includes, in order of progression: 1) Peripheral Autonomic Neuropathy (PAN), 2) Advanced Autonomic Dysfunction (AAD), and 3) Cardiovascular Autonomic Neuropathy (CAN). AAD is similar to Diabetic Autonomic Neuropathy (DAN) but independent of the other complications and symptoms associated with type 2 Diabetes Mellitus and the etiology is different. DAN and CAN have been well described [2,3]. Symptoms of autonomic neuropathy are recognized to increase morbidity and mortality in all patients with diseases known to also increase cardiovascular risk, including: 1) low ejection fraction [4,5].2) decreased cardiac output [6]. 3) arrhythmias $[7,8] .4)$ cardiomyopathies $[9,10]$ including Chronic Heart Failure [11]. 5) arteriosclerosis including coronary arteriosclerosis (Angina or Coronary Artery Disease) [12]. 6) silent myocardial infarction, sudden cardiac death [13]. 7) diabetes [2,3] and others [14,15]. These comorbidities, including the major adverse cardiovascular events (MACE), justify the need for improvement over standard therapies. $\mathrm{P} \& S$ monitoring provides more information to further improve patient outcomes.

\section{Methods}

A database of 3670 consecutive, serial patients [16] was followed for four years in a large cardiology practice drawing from both urban and suburban populations. Patients were aggressively managed according to clinical practice standards. While P\&S Monitoring facilitates reading $\mathrm{P} \& S$ responses in the presence of arrhythmia [17], to permit comparison with standard HRV responses, 943 were omitted due to high burden of ectopy. The remaining 2727 patients (50.5\% Female; average age $=57$ years; age range: 12 to 100 years) were followed with more than one P\&S Monitoring assessment over four (4) years (an average of 3.3 tests per patient). The mean time between P\&S tests is 442.7 days. Patients carry a diagnosis of cardiovascular disease (CVD) or a disease with a risk for CVD, such as hypertension (32.7\%), heart failure (35.2\%), history of MI (16.2\%), type 2 diabetes (36.2), renal disease (17\%), or COPD (8.7\%). Patients are on standard therapy [16]. Patients within this cohort also diagnosed with autonomic neuropathy $(65.6 \%)$ were prescribed either standard $\beta$ B therapy $(57.1 \%$ of those with autonomic neuropathy) or dose equivalent, or lower, Carvedilol titrated from 3.125 $\mathrm{mg}$ bid (42.9\% of those with autonomic neuropathy).

P\&S function was assessed noninvasively using the ANSAR Medical Technologies, Inc. (Philadelphia, PA) software (ANX 3.0 autonomic function monitor) which computes simultaneous, independent measures of $\mathrm{P} \& \mathrm{~S}$ activity based on continuous, time-

${ }^{\star}$ Correspondence to: Nicholas L DePace, Hahnemann Hospital, Drexel University College of Medicine, Franklin Cardiovascular Associates, PA., 438 Ganttown Rd., Ste. B8-B9, Sewell, NJ, 08080, USA, E-mail: dovetech@erols.com

Received: October 01, 2018; Accepted: October 18, 2018; Published: October 25,2018 

carvedilol

frequency analysis of heart rate variability (HRV) with concurrent, continuous time-frequency analysis of respiratory activity (RA) [18]. Clinical data collected include Heart Rate (HR), BP, Sympathovagal Balance (SB), AAD, which is similar to DAN, CAN, Ejection Fraction (EF) [19], Framingham Risk Score (FRS) [20] and Reynolds Risk Score (RRS: "Model B") [21]. SB is the ratio of resting sympathetic (S) activity $(\mathrm{LFa}$, the S-measure: Low Frequency area) to resting P-activity (RFa, the P-measure: Respiratory Frequency area) [16]. SB is an average of ratios ( $\mathrm{LFa} / \mathrm{RFa})$, not the ratio of averages. Normal SB ranges from 0.4 to 3.0 (unitless) [17].

$\mathrm{AAD}$, including $\mathrm{DAN}$, is present if either or both resting $\mathrm{LFa}$ or resting $\mathrm{RFa}$ are less than $0.5 \mathrm{bpm}^{2}$. CAN is present if $\mathrm{RFa}$ is less than $0.1 \mathrm{bpm}^{2}$. AAD, including DAN, indicates risk of morbidity [2]. CAN indicates risk of mortality [3]. AAD (or DAN) with abnormal SB indicates high morbidity risk. AAD (or DAN) with normal SB indicates normal morbidity risk. CAN with high SB (indicating S-excess, SE) indicates high morbidity and mortality risk, including MACE [22]. CAN with low SB (indicating P-excess, PE, associated with depression) indicates elevated morbidity and mortality risk [12] (e.g., depression and "Broken Heart Syndrome"). CAN with normal SB indicates normal risk. Of the patients diagnosed with autonomic neuropathy with abnormal SB, patients with AAD (or DAN) accounted for $52.6 \%$ overall, or $80.2 \%$ of the autonomic dysfunction sub-population. Patients with CAN accounted for $13.0 \%$ of the population or $19.8 \%$ of the autonomic dysfunction sub-population. Normalizing SB treats AAD (DAN) and CAN and minimizes risk [1].

The clinical study employed to determine P\&S activity includes four well-known autonomic challenges, separated by resting baseline periods. These six periods are labeled in the figures as: A) resting Baseline, B) Deep Breathing, C) baseline, D) Valsalva maneuvers, E) baseline, and F) Stand (postural change). The stand challenge is a postural change challenge, which is equivalent tilt-testing [24]. The stand challenge is a physiologic activity and therefore inherently safer and more comfortable for the patient, arguably leading to more reliable results [16]. The stand challenge enables autonomic testing to be performed in smaller clinics, and in shorter time periods. From a safety point of view, the independent measures of P\&S activity obviate the need for overt symptoms to be demonstrated, thereby inherently improving the safety of the study.

HRV-alone analyses compute mixed measures of P\&S activity. For example, spectral HRV analyses result in a Low Frequency (LF) and a High Frequency (HF) term $[25,26]$. LF is a mix of both P\&S activity unless the subject's breathing rate is greater than about 13 breaths per minute [25-27]. HF is a broad-band term [25-27], more than twice as broad as the known Parasympathetic frequency range [28-33]. Therefore, even if the subject's breathing rate is $>13$ breathes $/ \mathrm{min}$, the HF term is mixed with noise, including harmonics. Both LF \& HF require assumption and approximation to specify $\mathrm{P} \& \mathrm{~S}$ activity.

To eliminate the need for assumption and approximation required by LF \& HF, independent spectral analyses of RA are added to spectral analyses of HRV. This second independent P\&S measure (RA) satisfies the algebraic requirement for a system with two independent components, fully characterizing the system, eliminating the need for assumption and approximation. Wavelet analysis eliminates the timefrequency approximations required by Fourier Transforms and enables a significantly shorter data collection time to compute P\&S activity. This enables autonomic transients and the dynamic activity of P\&S interactions to be captured and analyzed. The resulting P\&S terms are Respiratory Frequency area (RFa) and Low Frequency area (LFa), respectively, and Sympathovagal Balance ( $\mathrm{SB}=\mathrm{LFa} / \mathrm{RFa}$ ) is computed as a true ratio of independent parameters [27].

Tilt-table testing is currently accepted as a standard of autonomic testing, and can be useful for certain diagnoses, such as cardiogenic syncope. However, there are numerous other diagnoses associated with autonomic testing for which tilt-table testing does not distinguish. Beat-To-Beat Blood Pressure (bbtBP, or in simpler form, Pulse Wave Velocity) is also a standard for autonomic testing. While btbBP is simpler to implement than tilt-table, it is often used in conjunction with tilt-table and, like tilt-table results, requires waveform assessment. Both are qualitative, subjective assessments, even in the hands of experts, and is only capable of analysis on the macroscopic level, once, waveforms are no longer visible to the human eye, no further analyses are possible. Furthermore, increases P- (or Vagal) activity improves the appearance of HRV (via improved respiratory sinus arrhythmia) or btbBP, to the point of not being able to visually differentiate normal from excessive before arrhythmia presents. This is the reason behind the lack of an upper bound to the normal range for HRV-based P-activity. ANSAR technology is quantitative and objective and utilizes frequency domain analyses beyond the visual range.

This is an observational study. Patient testing and clinical outcomes measures were collected as an authorized part of the subjects' care and treatment given their clinical history. All data were handled according to HIPPA regulations. Data were analyzed, statistically, with SPSS v 22.0 , with the null hypothesis indicating significance at $\mathrm{p} \leq 0.0500$.

\section{Results}

Two sub-populations were analyzed: one diagnosed with AAD / DAN and the other diagnosed with CAN. Due to the aggressive nature of therapy in these populations, BMI, HR, and BP differences are significant between the two sub-populations. For example, patients' BMI at baseline was and average 0.7 \# /in ${ }^{2}$ less at follow-up (29.8 \# / in $^{2}$ ). (Tables 1-3)

Within the cohort, $65.6 \%$ are diagnosed with some form of autonomic neuropathy (AAD or DAN, or CAN) with abnormal SB, by both symptoms and P\&S Monitoring. Within the autonomic neuropathy sub-population, $80.2 \%$ are diagnosed with AAD and $19.2 \%$ are diagnosed with CAN. There is no significant statistical difference between the AAD and CAN sub-populations regarding BMI, resting $\mathrm{HR}$, or resting BP ( $\mathrm{p}>0.12$, (Table 1$)$. Resting P\&S measures (LFa, RFa, and $\mathrm{SB}$ ) and risk factor measures (EF, FRS, and RRS) are significantly different $(p<0.04$, Table 1). Diabetes and depression diagnoses are not significantly different between the AAD and CAN sub-populations ( $>0.25$, Table 2). Patients with history of MI, stents, alcohol abuse (Table 2), and Carotid Stenosis (Table 3) are also not statistically different between the two sub-populations $(p>0.39)$. The greater number of patients diagnosed with CAN and also with CAD, RD, HF, or history of Ischemia, is statistically significant $(p<0.04$, Table 2$)$. Except for CRP ( $\mathrm{p}=0.05)$, none of the blood test results are significantly different between AAD and CAN ( $\mathrm{p}>0.07)$.

Irrespective of AAD or CAN, the clinical goal is to normalize $\mathrm{SB}$ to minimize risks. As a result, the difference between the AAD and CAN responses to $\beta B$ or Carvedilol therapy was not statistically significant ( $>00.82$ ). Table 4 presents the changes in HR \& BP, resting P\&S activity, and risk factors at baseline and follow-up, in response the two adrenergic antagonist therapies. Table 4 only presents the correlations between the two therapy populations for the baseline versus follow-up Carvedilol sub-populations. The rest of the possible sub-population comparisons did not demonstrate statistical significance $(\mathrm{p}>0.10)$. 

carvedilol

Within this aggressively treated cohort, patients HRs are very similar whether on standard $\beta B$ therapy or Carvedilol. Upon follow-up, there is a small (approximately $2 \mathrm{bpm}$ ) decrease in $\mathrm{HR}$ regardless of type of blockade therapy titrated in establishing and maintaining normal SB. The difference within the Carvedilol sub-population is not statistically significant $(\mathrm{p}=0.39$, Table 4$)$. Similarly, patients BPs are very similar at baseline. Upon follow-up, there is a small, but not significant $(\mathrm{p}=0.68$, Table 4) increase in BP regardless of type of blockade therapy titrated. Likewise, the resting S-responses (LFa) are not significant $(p=0.052$, between baseline and follow-up (Table 4).

Continuing with the data from Table 4, the resting P-responses (RFa) are significantly different between the baseline and follow-up responses to Carvedilol $(\mathrm{p}=0.006)$, but not in response to standard $\beta B$ therapy $(p=0.85)$. This significance is reflected in the $S B$ responses between baseline and follow-up. The response with Carvedilol is significant $(\mathrm{p}=0.02)$. The response with standard $\beta \mathrm{B}$ therapy is not $(\mathrm{p}=0.10)$. In response to standard $\beta B$ therapy, there is a small decrease in EF upon follow-up; however, in response to Carvedilol there is a significant increase in EF $(\mathrm{p}=0.01)$. The two risk scores are decreased in response to either adrenergic blockade; however, they are only statistically significantly decreased in response to Carvedilol: FRS, $\mathrm{p}=0.04 ;$ RRS, $\mathrm{p}=0.04$.

\section{Discussion}

Within this aggressively treated cardiology cohort, AAD and CAN patients are well matched in BMI, resting HR and BP (Table 1), comorbidities (Table 2), and blood test results (Table 3). The differences in comorbidities (Coronary Artery Disease, Renal Insufficiency, Heart Failure, and Ischemia) are as expected, given the known correlation between the progression of CVD and autonomic neuropathy. From reported blood test results, only the inflammatory marker CRP is significantly different when comparing AAD with CAN. This is not unexpected; inflammation is significantly greater in patients with CAN than with AAD. Inflammation has been correlated with degree of autonomic neuropathy (a relative S-excess) [34].

The fact that $\mathrm{P} \& S$ measures and $\mathrm{EF}$ are reduced, and the risk scores are higher in patients demonstrating CAN as compared with AAD is also expected. Reduced EF and higher risk scores are correlated with increased mortality risk, as is CAN. AAD indicates increased morbidity risk, whereas CAN (a more severe form of autonomic neuropathy with its lower, absolute, autonomic levels) indicates increased morbidity and mortality risk. Herein lies where SB is more important than absolute levels. Normal balance helps to normalize risk, even if it means reducing the activity in one branch to balance it with the other.

In cases of low SB where the absolute, resting P-level is significantly greater than the S-level, (sub-clinical) depression is possible. This may be the case, even when the absolute P\&S levels are low as is often demonstrated by patients demonstrating CAN. Depression is known to elevate mortality risk [23]. CAN already indicates elevated mortality risk. Therefore, low SB (e.g., sub-clinical depression) may exacerbate CAN and associated mortality risk. On the other hand, if SB is too high, then high mortality risk is indicated [22], or exacerbated in the case

Table 1. Baseline testing results: BMI and clinical measures of HR, BP, P\&S measures, EF, and risk scores

\begin{tabular}{|c|c|c|c|c|c|c|c|c|c|}
\hline Population & BMI & mHR & BP & LFa & RFa & SB & EF & FRS & RRS \\
\hline AAD & 31.53 & 69.2 & $123 / 68$ & 0.82 & 0.42 & 3.21 & 62.2 & 18.8 \\
\hline CAN & 31.26 & 68.4 & $125 / 69$ & 0.17 & 0.05 & 3.02 & 46.0 \\
\hline $\mathbf{p}$ & 0.67 & 0.12 & 0.20 & 0.02 & 0.03 & 0.03 & 0.02 \\
\hline
\end{tabular}

AAD: Advanced Autonomic Dysfunction (or DAN, if diabetic); BMI: Body Mass Index (\#/in²); BP: Blood Pressure (mmHg); CAN: Cardiovascular Autonomic Neuropathy; EF: Ejection Fraction (\%); FRS: Framingham Risk Score (\%); LFa: Low Frequency area, the Sympathetic measure; mHR: mean Heart Rate; RFa: Respiratory Frequency area, the Parasympathetic measure; RRS: Reynolds Risk Score (\%); p: statistical significance

Table 2. Baseline testing results: comorbidities by percent of sub-populations

\begin{tabular}{|c|c|c|c|c|c|c|c|c|c|}
\hline Population & DM2 & CAD & RI & HF & Isch & MI & Dep & EtOH & Stents \\
\hline AAD (\%) & 29 & 17 & 8 & 5 & 1 & 15 & 8 \\
\hline CAN (\%) & 28 & 34 & 19 & 10 & 12 & 8 & 11 \\
\hline p & 0.59 & 0.04 & 0.02 & 0.03 & 0.04 & 0.60 & 0.25 \\
\hline
\end{tabular}

EtOH: Alcohol abuse; CAD: Coronary Artery Disease; Dep: Depression; DM2: type 2 Diabetes Mellitus; HF: Heart Failure; Isch: history of cardiac Ischemia; MI: history of Myocardial Infarction; Stents: one or more stents implanted; RI: Renal Insufficiency; see Table 1 for additional abbreviations

Table 3. Baseline testing results: average blood test results by sub-population, and percent of sub-population with Carotid Stenosis

\begin{tabular}{|c|c|c|c|c|c|c|c|c|c|}
\hline Population & HDL & LDL & Tri & A1c & CRP & BNP & Creat & Ca++ & CS \\
\hline AAD & 35.4 & 163.7 & 156.7 & 5.5 & 2.57 & 219 & 1.05 & 8.0 \\
\hline CAN & 34.3 & 161.7 & 135.0 & 4.8 & 3.12 & 367 & 0.84 & 8.5 \\
\hline $\mathbf{p}$ & 0.72 & 0.94 & 0.18 & 0.59 & 0.05 & 0.07 & 0.12 \\
\hline
\end{tabular}

Alc: hemoglobin Alc (\%); BNP: B-type Natriuretic Peptide (pg/mL); Ca++: blood Calcium (mg/dL); CS: Carotid Stenosis (\%); Creat: Creatinine (mg/dL); CRP: C-Reactive Proteins (mg/dL); HDL: High Density Lipoproteins (mg/dL); LDL: Low Density Lipoproteins (mg/dL); Tri: Triglycerides (mg/dL); see Table 1 for additional abbreviations

Table 4. Changes in HR \& BP, resting P\&S activity, and risk factors in the autonomic neuropathy sub-population at baseline and follow-up, in response $\beta B$ and Carvedilol therapies. Only the baseline versus follow-up responses for the Carvedilol sub-population demonstrated any statistical significance

\begin{tabular}{|c|c|c|c|c|c|c|c|c|c|}
\hline Test & Population & mHR & BP & LFa & RFA & SB & $\mathbf{E F}$ & FRS & RRS \\
\hline \multirow{2}{*}{ Baseline } & $\boldsymbol{\beta B}$ & 69.4 & $125 / 73$ & 0.55 & 0.06 & 9.09 & 48.5 & 18.7 & 19.0 \\
\hline & Carvedilol & 69.3 & $126 / 71$ & 0.61 & 0.09 & 6.48 & 46.9 & 18.3 & 19.9 \\
\hline \multirow{2}{*}{ Follow-up } & $\beta B$ & 67.5 & $128 / 78$ & 0.53 & 0.07 & 7.35 & 41.7 & 17.7 & 17.6 \\
\hline & Carvedilol & 67.4 & $127 / 72$ & 0.51 & 0.29 & 1.59 & 60.1 & 15.1 & 15.8 \\
\hline $\begin{array}{l}\text { Bx v F/U, } \\
\text { Carvedilol* }\end{array}$ & $\mathbf{p}$ & 0.39 & 0.68 & 0.05 & 0.006 & 0.02 & 0.01 & 0.04 & 0.04 \\
\hline
\end{tabular}

Bx: Baseline; F/U: Follow-up; $\beta$ B: Beta-blocker; see Table 1 for additional abbreviations. *All correlations, other than the responses to Carvedilol are not significant ( $\mathrm{p}>0.10$ ) 

carvedilol

of CAN, due to the effects of a relative S-excess (relative to P-activity) regardless of the absolute S-level.

The follow-up responses to standard $\beta \mathrm{B}$ therapy and Carvedilol therapy involve the autonomic nervous system. Both types of therapy involve adrenergic antagonism, thereby reducing S-activity. HR and $\mathrm{BP}$ are driven by S-activity. Therefore, the similarities between the maintenance of lower S-activity and lower HR and BP, in this aggressively treated cardiology cohort, are not unexpected, given that S-blockade (adrenergic antagonists) primarily treats excess S-activity. Apparently, however, both types of therapy do not involve P-effects.

Carvedilol does cause statistically significant differences between follow-up P-responses to standard $\beta \mathrm{B}$ therapy and Carvedilol therapy. The standard $\beta \mathrm{B}$ therapies used in this study do not. Carvedilol appears to have a parasympathomimetic effect, either (or perhaps both) due to its (1) antioxidant and anti-inflammatory effects [35-37], and (2) vasodilatory, $\alpha$-adrenergic antagonist, effects (reducing both systolic and diastolic BP) [38], indirectly enhancing P-activity. P-insufficiency differentiates CAN from other forms of autonomic neuropathy or dysfunction. CAN is a more advanced form of dysfunction than $\mathrm{AAD}$ or DAN. CAN is associated with lower EF and greater risk scores. Therefore, it is reasonable to hypothesize that also treating P-insufficiency would improve patient outcomes over standard $\beta \mathrm{B}$ therapy, perhaps by slowing or reversing the progression of heart failure, and /or risk of sudden cardiac death or other MACEs, even in an already aggressively treated cardiology cohort.

\section{Conclusion}

Adrenergic blockers establish and maintain lower $\mathrm{HR}$ and $\mathrm{BP}$ by reducing $\mathrm{S}$-activity as measured by a reduction in SB. In this way, they also treat autonomic neuropathy (AAD, DAN, or CAN), and help to moderate risk as indicated by lower EF and risk scores. Furthermore, normalizing SB optimizes this moderation. This is well known. Carvedilol, a combined drug, seems to further improve patient outcomes through also improving P-activity. Carvedilol, as compared with standard $\beta B$ therapy improved outcomes as measured by $P \& S$ measures, BP, HR, EF, Framingham risk score, and Reynolds risk score. Within this cohort, Carvedilol normalized SB, whereas standard $\beta B$ therapy reduced SB. Normalizing SB with Carvedilol within this cohort increases EF and further reduces risk, as indicated by the Framingham or Reynolds methods.

\section{Conflicts of interest}

Dr. Vinik has been paid honoraria for lecturing on behalf of ANSAR Medical Technologies, Inc. Otherwise, the authors have no conflicts of interest in this study.

\section{References}

1. Bloom HL, Vinik AI, Colombo J (2014) Differential effects of adrenergic antagonists (carvedilol vs. metoprolol) on parasympathetic and sympathetic activity: A comparison of clinical results. Heart Int 9: 15-21. [Crossref]

2. Vinik AI, Maser RE, Nakave AA (2007) Diabetic cardiovascular autonomic nerve dysfunction. Endocr Pract 2-9.

3. Vinik A, Ziegler D (2007) Diabetic cardiovascular autonomic neuropathy. Circulation 115: 387-397.

4. DePace NL, Bateman JA, Yayac M, Oh J, Siddique M, et al. (2018) Improved Patient Outcomes by Normalizing Sympathovagal Balance: Differentiating Syncope-Precise Subtype Differentiation Leads to Improved Outcomes. Cardiol Res Pract.

5. Nanavati SH, Bulgarelli RJ, Vazquez-Tanus J, Ghosh-Dastidar S, Colombo J, et al. (2010) Altered autonomic activity with atrial fibrillation as demonstrated by noninvasive autonomic monitoring. US Cardio 7: 47-50.
6. Malik, M (1996) The Task Force of the European Society of Cardiology and the North American Society of Pacing and Electrophysiology. Heart rate variability, standards of measurement, physiological interpretation, and clinical use. Circulation 93: 10431065. [Crossref]

7. Malik M (1996) The Task Force of the European Society of Cardiology and the North American Society of Pacing and Electrophysiology. Heart rate variability, standards of measurement, physiological interpretation, and clinical use. Circulation 17: 354-381. [Crossref]

8. Colombo J, Arora RR, DePace NL, Vinik AI (2014) Clinical Autonomic Dysfunction: Measurement, Indications, Therapies, and Outcomes. Springer.

9. Bullinga JR, Alharethi R, Schram MS, Bristow MR, Gilbert EM (2005) Changes in heart rate variability are correlated to hemodynamic improvement with chronic CARVEDILOL therapy in heart failure. J Card Fail 11: 693-699. [Crossref]

10. Fatoni C, Raffa S, Regoli F, Giraldi F, La Rovere MT, et al. (2005) Cardiac resynchronization therapy improves heart rate profile and heart rate variability of patients with moderate to severe heart failure. $J$ Am Coll Cardiol 46: 1875-1882. [Crossref]

11. Fathizadeh P, Shoemaker WC, Wo CC, Colombo J (2004) Autonomic activity in trauma patients based on variability of heart rate and respiratory rate. Crit Care Med 32: 13001305. [Crossref]

12. Curtis BM, O'Keefe JH Jr (2002) Autonomic tone as a cardiovascular risk factor: the dangers of chronic fight or flight. Mayo Clin Proc 77: 45-54. [Crossref]

13. Lichtman JH, Bigger JT Jr, Blumenthal JA, Frasure-Smith N, Kaufmann PG, et al (2008) Depression and coronary heart disease recommendations for screening, referral, and treatment: A science advisory from the American Heart Association Prevention Committee of the Council on Cardiovascular Nursing, Council on Clinical Cardiology, Council on Epidemiology and Prevention, and Interdisciplinary Council on Quality of Care and Outcomes Research: Endorsed by the American Psychiatric Association. Circulation 118: 1768-1775. [Crossref]

14. Chen PS, Chou CC, Tan AY, Zhou S, Fishbein MC, et al. (2006) The mechanisms of atrial fibrillation. J Cardiovasc Electrophysiol 3: S2-7. [Crossref]

15. Copie X, Lamaison D, Salvador M, Sadoul N, DaCosta A, et al. (2003) Heart rate variability before ventricular arrhythmias in patients with coronary artery disease and an implantable cardioverter defibrillator. Ann Noninvasive Electrocardiol 8: 179-184. [Crossref]

16. Alter P, Grimm W, Vollrath A, Czerny F, Maisch B (2006) Heart rate variability in patients with cardiac hypertrophy--relation to left ventricular mass and etiology. $\mathrm{Am}$ Heart J 151: 829-836. [Crossref]

17. Debono M, Cachia E (2007) The impact of cardiovascular autonomic neuropathy in diabetes: is it associated with left ventricular dysfunction? Auton Neurosci 132: 1-7. [Crossref]

18. Just H (1991) Peripheral adaptations in congestive heart failure: a review. Am J Med 90 23S-26S. [Crossref]

19. Manfrini O, Morgagni G, Pizzi C, Fontana F, Bugiardini R (2004) Changes in autonomic nervous system activity: spontaneous versus balloon-induced myocardial ischaemia. Eur Heart $J$ 25: 1502-1508. [Crossref]

20. Clarke BF, Ewing DJ, Campbell IW (1979) Diabetic autonomic neuropathy. Diabetologia 17: 195-212. [Crossref]

21. DePace NL, Mears JP, Yayac M, Colombo J (2014) Cardiac autonomic testing and diagnosing heart disease. "A clinical perspective." Heart International 9: 37-44. [Crossref]

22. DePace NL, Mears JP, Yayac M, Colombo J (2014) Cardiac autonomic testing and treating heart disease. "A clinical perspective." Heart International 9: 45-52. [Crossref]

23. Vinik AI, Bloom HL, Colombo J (2014) Differential effects of adrenergic antagonists (carvedilol vs. metoprolol) on parasympathetic and sympathetic activity: A comparison of measures. Heart Int 9: 7-14. [Crossref]

24. Teichholz LE, Kreulen T, Herman MV, Gorlin R (1976) Problems in echocardiographic volume determinations: echocardiographic-angiographic correlations in the presence of absence of asynergy. Am J Cardiol 37: 7-11. [Crossref]

25. D'Agostino RB Sr, Vasan RS, Pencina MJ, Wolf PA, Cobain M, et al. (2008) General cardiovascular risk profile for use in primary care: the Framingham Heart Study. Circulation 117: 743-753. [Crossref]

26. Ridker PM, Buring JE, Rifai N, Cook NR (2007) Development and validation of improved algorithms for the assessment of global cardiovascular risk in women: the Reynolds Risk Score. JAMA 297: 611-619. [Crossref] 
DePace NL (2018) Improved patient outcomes by normalizing sympathovagal balance as measured by parasympathetic and sympathetic monitoring: the benefits of carvedilol

27. Bloomfield DM, Kaufman ES, Bigger JT Jr, Fleiss J, Rolnitzky L, et al. (1997) Passive head-up tilt and actively standing up produce similar overall changes in autonomic balance. Am Heart J 134: 316-320.

28. Akselrod S, Gordon S, Ubel FA, Shannon DC, Berger AC (1981) Power spectrum analysis of heart rate fluctuations: a quantitative probe of beat-to- beat cardiovascular control. Science 213: 213-220.

29. Akselrod S, Gordon D, Madwed JB, Snidman NC, Shannon DC, et al. (1985) Hemodynamic regulation: investigation by spectra analysis. Am J Physiol 249: H86775. [Crossref]

30. Akselrod S, Eliash S, Oz O, Cohen S (1987) Hemodynamic regulation in SHR: investigation by spectral analysis. Am J Physiol 253: H176-183. [Crossref]

31. Akselrod S (1988) Spectral analysis of fluctuations in cardiovascular parameters: a quantitative tool for the investigation of autonomic control. Trends Pharmacol Sci 9 : 6-9. [Crossref]

32. Aysin B, Aysin E (2006) Effect of respiration in heart rate variability (HRV) analysis. Conf Proc IEEE Eng Med Biol Soc 1: 1776-1779. [Crossref]
33. Aysin B, Colombo J, Aysin E (2007) Comparison of HRV analysis methods during orthostatic challenge: HRV with respiration or without? Conf Proc IEEE Eng Med Biol Soc 2007: 5047-5050. [Crossref]

34. Vinik AI, Erbas T, Casellini CM (2013) Diabetic cardiac autonomic neuropathy, inflammation and cardiovascular disease. J Diabetes Investig 4: 4-18. [Crossref]

35. Arumanayagam M, Chan S, Tong S, Sanderson JE (2001) Antioxidant properties of carvedilol and metoprolol in heart failure: a double-blind randomized controlled trial. $J$ Cardiovasc Pharmacol 37: 48-54. [Crossref]

36. Dandona P, Ghanim H, Brooks DP (2007) Antioxidant activity of carvedilol in cardiovascular disease. J Hypertens 25: 731-741. [Crossref]

37. Ayashi S, Assareh AR, Jalali MT, Olapour S, Yaghooti H (2016) Role of antioxidan property of carvedilol in mild to moderate hypertensive patients: A prospective openlabel study. Indian J Pharm 48: 372-376. [Crossref]

38. Sponer G, Strein K, Bartsch W, Müller-Beckmann B (1992) Vasodilatory action of carvedilol. J Cardiovasc Pharmacol 19 Suppl 1: S5-11. [Crossref]

Copyright: $\mathbb{C} 2018$ DePace NL. This is an open-access article distributed under the terms of the Creative Commons Attribution License, which permits unrestricted use, distribution, and reproduction in any medium, provided the original author and source are credited. 